\title{
A Methodical Approach to Interpreting the Platelet Parameters of the Complete Blood Count
}

\section{KATHY DOIG, MICHELLE BUTINA}

\section{LEARNING OBJECTIVES}

1. List the platelet parameters of a complete blood count $(\mathrm{CBC})$ and additional special tests of platelet age.

2. Describe the principle of analysis for each of the platelet parameters of the CBC.

3. Describe the steps for interpretation of the platelet values on a CBC.

4. Recognize results consistent with spurious platelet values and recommend procedures to correct them.

5. Given $\mathrm{CBC}$ results, apply a methodical approach to thoroughly interpret each platelet value using the diagnostic heuristic provided below.

\section{ABSTRACT}

Consistent use of a methodical approach to interpreting complete blood count (CBC) results can help detect spurious results that require remedy before results are reported. It can also help ensure that no clinically important information is overlooked during diagnostic interpretation of the results. The steps for interpreting the platelet portion of the $\mathrm{CBC}$ are:

1. Interpret the total platelet count relative to the reference interval.

2. Examine the mean platelet volume and compare to the reference interval.

3. Examine the platelet distribution width and compare to the reference interval.

4. Examine the platelet distribution histogram for evidence of interferences. Take steps to correct these and reassess Steps 1-3 based on the revised results.

5. Examine the immature platelet fraction and compare to the reference interval.

6. Correlate the numerical parameters with platelet morphology.

7. Correlate platelet results with red blood and white blood cell parameters and conduct clinical assessment of the patient's values.
Explanations for conducting the evaluations are provided and the steps are applied to example cases to demonstrate the approach to interpreting the platelet parameters of the CBC.

ABBREVIATIONS: CBC - complete blood count, rbcred blood cell, RBC-red blood cell count, MCV-mean cell volume, plt-platelet, PLT-platelet count, RDW-red blood cell distribution width, RNA-ribonucleic acid, wbc-white blood cell, $\mu \mathrm{L}$-microliter

INDEX TERMS: Platelet counts, platelet number, mean platelet volume

Clin Lab Sci 2017;30(3):194

Kathy Doig, PhD, MLS(ASCP) $)^{C M} S H(A S C P)^{C M}$, Professor Emeritus, Biomedical Laboratory Diagnostics Program, Michigan State University, E. Lansing, MI

Michelle Butina, PhD, MLS(ASCP) ${ }^{C M}$, Associate Professor Medical Laboratory Science Program University of Kentucky College of Health Sciences Lexington, $K Y$

Address for Correspondence: Kathy Doig, PhD, $M L S(A S C P)^{C M} S H(A S C P)^{C M}, 354$ Farm Lane, Rm. N322, E. Lansing, $M I$ 48824, 517-353-1985, doig@msu.edu

\section{INTRODUCTION}

The complete blood count (CBC) is a set of analyses plus derived or calculated values for the formed elements of blood: red blood cells (rbcs), white blood cells (wbcs), and platelets (plts). For each element there is a group of parameters whose results should be interpreted in relation to the others in that group. Ultimately, results must be assessed across the groups to develop the full clinical significance of the results, which is beyond the scope of this article. This article will focus on the common plt parameters and present a heuristic to guide results interpretation within that group of results. A heuristic is an approach to problem solving; in this case, 
it is a set of steps to methodically think through platelet values interpretation. As a result, no value is overlooked and each is assessed in the context of the others.

The interpretation of laboratory results by laboratorians incorporates two levels of analysis. The first is assessment of the validity of the results to answer the question, "Can these results be reported?" Key operators must program instruments to detect invalid results or to confirm the instrument detection limits. Effectively, the key operator is programming the instrument to emulate an interpretive heuristic, like this one, to detect invalid results. And though modern instruments are then able to provide flags that alert operators to possible invalid results, flags should be used as guides. Some invalid results may escape detection by instruments and require a careful assessment by the operator. Furthermore, the instrument is not able to incorporate non-instrument results, such as blood film morphology, that may be critical to detection of spurious instrument results.

The second level of results analysis is clinical significance and whether subsequent actions like reflex testing, critical values procedures, or notifications to other departments are warranted. The literature frequently contains algorithms that are focused on the diagnostic aspects of test interpretation. However, the incorporation of the validation aspect is what distinguishes this heuristic.

The heuristic presented here is a series of steps that should be completed to ensure a thorough assessment of platelet-related $\mathrm{CBC}$ values. The recommended sequence is logical, but not fixed as long as a thorough review is conducted. It may be especially valuable to laboratory science students, pathology residents, or those who work infrequently in hematology.

All plt parameters discussed here may not be available on each manufacturer's instrument. Some available parameters may not yet be approved by the Food and Drug Administration (FDA), and thus not reportable. Such "research" parameters may be useful to operators for quality assurance checks. Institutions vary on whether the full suite of FDA-approved platelet parameters available on their hematology analyzers will be reported. However, even if FDA-approved parameters are not reported, they may provide important information for instrument operators in determining whether the platelet values are accurate.
The sequencing of the steps below is not necessarily fixed. But they comprise a thorough assessment and all steps should be conducted. The platelet portions of a CBC included here are, the platelet count (PLT), the mean platelet volume (MPV), the platelet distribution width (PDW) and the associated histogram, the immature platelet fraction (IPF), and platelet morphology. The steps for interpreting the platelet portion of the $\mathrm{CBC}$ are provided in Table 1.

\footnotetext{
Table 1. The steps for interpreting the platelet portion of a complete blood count.

1. Interpret the total platelet count relative to the reference interval.

2. Examine the MPV and compare to the reference interval.

3. Examine the PDW and compare to the reference interval.

4. Examine the platelet distribution histogram for evidence of interferences. Take steps to correct these and reassess Steps 1-3 based on the revised results.

5. Examine the IPF and compare to the reference interval.

6. Correlate the numerical parameters with platelet morphology.

7. Correlate platelet results with $\mathrm{rbc}$ and wbc parameters and conduct clinical assessment of the patient's values.
}

\section{Using the Steps to Interpret the Platelet Parameters of a CBC}

Step 1. Interpret the total platelet count relative to the reference interval.

An increase in platelets above the reference interval is called thrombocytosis while a decrease is called thrombocytopenia. Causes of falsely high values include the false increase due to fragmented rbcs, which is discussed below. However, a false decrease can occur in some patients when platelets adhere to neutrophils in a process called platelet satellitosis or satellitism ${ }^{1}$ that is caused by a reaction between the patient's platelets and ethylenediaminetetraacetic acid (EDTA) anticoagulant. For any patient with a previously unrecognized thrombocytopenia, examination of a peripheral blood film should be conducted to detect possible satellitosis. An accurate platelet count is obtained by recollecting a sample in citrate (blue top tube) and analyzing the new sample with the instrument. ${ }^{2}$ The result must be multiplied by 1.1 to adjust for the dilution of the citrate anticoagulant. With an accurate platelet count, meaningful clinical assessments of thrombocytosis and thrombocytopenia can be made.

Step 2. Examine the MPV and compare to the reference 
interval.

The MPV is a measure of the size of the platelets, on average. Unlike volume changes for rbcs reflected by the mean cell volume (MCV), no specific terminology is associated with changes to the MPV. However, there are meaningful clinical associations, so recognizing changes from normal, particularly platelet enlargement that is reflected by an elevation of the MPV, are important. ${ }^{3}$ In instances when an automated platelet count is not available and the platelet count is determined by manual microscopic counting, the MPV will not be available and microscopic assessment of platelet size will be needed (see Step 6 below).

Step 3. Examine the PDW and compare to the reference interval.

The PDW is the platelet equivalent of the red blood cell distribution width (RDW). It is derived mathematically from the platelet frequency distribution and is a measure of the variability of platelet volumes (i.e. sizes). As a result, the value of the PDW can rise as platelets vary in size from small to large, but rarely will decrease as the variability in platelet size will be at its minimum in normal circumstances. While increases of the RDW correlate to the morphological recognition of anisocytosis for rbcs, there is no specific word for increased variation of platelet sizes. Though there is no terminology associated with broadening of the PDW, its recognition is important. Together with the MPV, it can point to the possibility of enlarged and giant platelets or even fragments of megakaryocyte cytoplasm, which are conditions with clinical significance. ${ }^{4}$ Like the MPV, a PDW will not be available for manual platelet counts, so microscopic assessment will be needed.

Step 4. Examine the platelet distribution histogram for evidence of interferences. Take steps to correct these and reassess Steps 1-3 based on the revised results.

A falsely elevated platelet count can occur on some instruments when other small particles, like fragmented rbcs, are counted in the same solution as the platelets. Instrument flags may alert the operator to this. In the instance that there is a high PDW, the platelet distribution histogram demonstrating a number of particles with a high volume, and there is a simultaneous RDW showing many very small rbcs, perhaps with a high take off (the curve does not originate at the origin of the $y$-axis), then the presence of rbc fragments contributing to the platelet count is likely. Examination of the blood film can confirm this conclusion. A manual platelet count may be needed in order to lyse interfering rbc fragments and resolve the false platelet count. Any assessment of the patient's platelets requires such resolution as a first step before clinical interpretation of the patient's other platelet parameters (Steps 1-3). While review of the platelet distribution histogram could be the first step of the process, relatively few samples will require this adjustment, thus the order of the steps as presented will be pertinent to most patient samples. The PDW histogram is another parameter that is not available with manual platelet counts.

Step 5. Examine the IPF and compare to the reference interval.

When platelets are released from the bone marrow, they are typically larger than platelets that have circulated longer. In addition, they contain more ribonucleic acid (RNA), which diminishes with aging. Thus, detecting the amount of RNA in platelets can be used to assess whether the bone marrow is responding to thrombocytopenia by producing young platelets. The percentage (or fraction) of the platelets that are immature constitutes the IPF. It is analogous to seeing an increase in reticulocytes during anemia.

The IPF is particularly useful in differentiating platelet consumption conditions from aplastic causes of thrombocytopenia. In the former, such as immune thrombocytopenia, peripheral destruction of older, smaller platelets is accompanied by a continued bone marrow release of younger and larger platelets (thus elevating the IPF). ${ }^{4,5}$ In aplasia, no large young platelets are released from the marrow, so the IPF is low. Corresponding changes to the shape of the platelet distribution histogram will be seen in each condition. Young platelets containing residual RNA can be stained and detected via flow cytometric methods. No specific terminology is used for changes to the IPF. There is no mechanism to assess the IPF with manual platelet counting.

Step 6. Correlate the numerical parameters with the reported platelet morphology. 
Normal platelet morphology is unmentioned in CBC reports. Morphology is described when it is abnormal and it should correlate to the numerical parameters. This provides a quality control check that the slide and the numerical values are for the same sample and a means to detect errors in the numerical values. An elevated MPV should correlate with the presence of larger than normal platelets on the blood film. Similarly, an elevated PDW should be reflected in variable sizes of platelets seen. An elevated IPF will typically correlate with a higher MPV. As noted above, microscopic examination of the platelets may be needed to detect situations in which the platelet count is not reliable.

Each clinical laboratory follows their protocol guiding the use of terminology, such as giant or enlarged, to describe oversized platelets. International recommendations are available. ${ }^{6}$ Additional descriptors are used for diminished granularity that can only be determined with microscopic examination, as they do not have a numerical counterpart. Platelets that exhibit dysplastic features such as blebbing should also be mentioned.

When the peripheral blood film is evaluated, the total platelet count should be estimated from the blood film ${ }^{2}$ and compared to the numerical value as a quality control check. A simple platelet estimate assumes a normal proportion of platelets to rbcs of about 1:20, thus multiplying the average number of platelets/100X field by $20 \times 10^{3} / \mu \mathrm{L}$ provides a good estimate of the platelet count. However, when the patient is anemic or erythrocythemic, this proportion may not be normal because the rbc portion of the ratio is altered. The estimate is improved in such instances, by the incorporating the total rbc count. This formula assumes approximately 250 rbcs are seen in one 100X high power field (hpf; Figure 1).

Estimated platelet count $\left(\times 10^{3} \mu \mathrm{L}\right)=$ Ave number of platelets/hpf $\mathrm{x}$ $\mathrm{RBC}\left(\mathrm{x} 10^{6} \mu \mathrm{L}\right) / 250 \mathrm{rbcs}$

Figure 1. The equation for estimating a platelet count is shown.

In instances where there are serial samples on a patient with known abnormalities, blood film examination need only be done periodically and should follow institutional protocol. Furthermore, in instances in which the platelet count has been performed manually, the morphological report has added significance since no numerical parameters will be available to indicate likely abnormalities in platelet size.

Step 7. Correlate platelet results with $\mathrm{rbc}$ and wbc parameters and conduct clinical assessment of the patient's values.

Having carefully assessed the full suite of platelet parameters and ensured their accuracy, the results can be assessed for clinical significance. Naturally, the platelet results must be considered along with the rbc and wbc values, since some clinical conditions will affect the others as well. Evidence of those may have been revealed in the platelet analysis, such as rbc hemolysis that interfered with the platelet count.

\section{Applying the Interpretive Steps to Case Examples}

Case 1. A 39-year-old female was referred for CBC testing by her family physician. Refer to Table 2 and Figure 2 for the platelet parameters on this patient.

\begin{tabular}{lll}
\hline \multicolumn{2}{l}{ Table 2. Platelet Parameter Results for Case 1. } \\
\hline Assay & Patient & Reference Interval \\
\hline Platelet count $\left(\times 10^{3} / \mu \mathrm{L}\right)$ & 713 & $150-450$ \\
MPV (fL) & 10 & $8-12$ \\
PDW (fL) & 13 & $9-15$ \\
Histogram & Refer to Figure 2. & Refer to Figure 2. \\
IPF (\%) & 5 & $0.5-7$ \\
Platelet Morphology & No abnormalities & Normal \\
& noted. \\
\hline
\end{tabular}

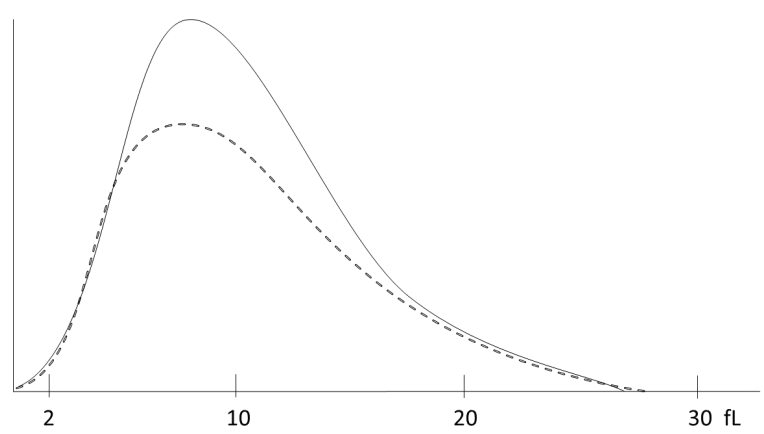

Figure 2. Platelet histogram for case study 1 . The dotted line represents a normal histogram. The solid line represents the patient histogram.

1. Interpret the total platelet count relative to the reference interval.

The initial platelet count was $713 \times 10^{3} / \mu \mathrm{L}$, thus thrombocytosis. 
2. Examine the MPV and compare to the reference interval.

The MPV was within the reference interval.

3. Examine the PDW and compare to the reference interval.

The PDW was within the reference interval.

4. Examine the platelet distribution histogram for evidence of interferences. Take steps to correct these and reassess Steps 1-3 based on the revised results.

The platelet distribution histogram was of normal shape but increased height, consistent with the thrombocytosis.

5. Examine the IPF and compare to the reference interval.

The IPF was within the reference interval.

6. Correlate the numerical parameters with platelet morphology.

No abnormalities of platelet morphology were noted which is consistent with the normal values of the platelet indices. A platelet estimate would need to encounter an average on the order of 55 platelets/100X field. When multiplied by $20 \times 10^{3} / \mu \mathrm{L}$, it would generate an estimated platelet count of $1100 \times 10^{3} / \mu \mathrm{L}$. However, since the patient was anemic (results not shown), the estimate would be improved by using the formula presented above. Assuming a rbc count (not shown) of $3.3 \times 10^{6} / \mu \mathrm{L}$, the resulting estimate would be $726 \times$ $10^{3} / \mu \mathrm{L}$, which is very close to the automated value. The peripheral blood film confirmed thrombocytosis and the accuracy of automated platelet count.

7. Correlate platelet results with $\mathrm{rbc}$ and wbc parameters and conduct clinical assessment of the patient's values.

This patient's significant thrombocytosis of normal platelets was part of a CBC (results not shown here) that included a slight leukocytosis with absolute neutrophilia, eosinophilia and basophilia. The $\mathrm{CBC}$ also demonstrated a normocytic, normochromic anemia. These findings would raise a suspicion of chronic myelogenous leukemia.

The family physician had noted pallor and splenomegaly, and with the $\mathrm{CBC}$ results was indeed concerned for leukemia, so she referred the patient to a hematology/oncology physician. The second physician ordered cytogenetic testing that demonstrated a $t(9 ; 22)$ on the bone marrow sample and revealed a BCR/ABL translocation which was confirmed by polymerase chain reaction. The bone marrow also demonstrated a high number of normal appearing megakaryocytes. The patient was diagnosed with chronic-phase chronic myelogenous leukemia.

Case 2. A 55-year-old male was referred by an orthopedic specialist for a CBC. The platelet results are provided in Table 3 and depicted in Figure 3.

\begin{tabular}{|c|c|c|}
\hline Assay & Patient & Reference Interval \\
\hline $\begin{array}{l}\text { Platelet count ( } \mathrm{x} \\
\left.10^{3} / \mu \mathrm{L}\right)\end{array}$ & 19 & $150-450$ \\
\hline MPV (fL) & 14 & $8-12$ \\
\hline PDW (fL) & 16 & $9-15$ \\
\hline Histogram & Refer to Figure 3. & Refer to Figure 3. \\
\hline IPF (\%) & 12 & $0.5-7 \%$ \\
\hline Platelet Morphology & $\begin{array}{l}\text { Few giant platelets } \\
\text { noted. }\end{array}$ & Normal \\
\hline
\end{tabular}

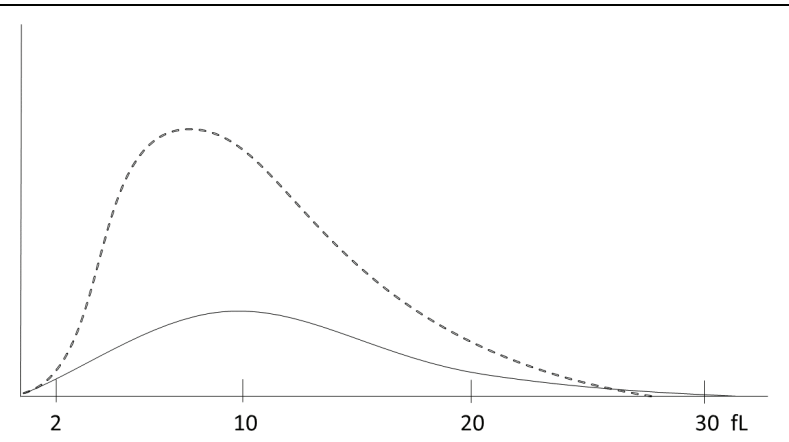

Figure 3. Platelet histogram for case study 2. The dotted line represents a normal histogram. The solid line represents the patient histogram.

1. Interpret the total platelet count relative to the reference interval.

The results indicated a platelet count below the reference interval, thus thrombocytopenia.

2. Examine the MPV and compare to the reference 
interval.

The MPV was elevated above the reference interval.

3. Examine the PDW and compare to the reference interval.

The PDW was elevated above the reference interval.

4. Examine the platelet distribution histogram for evidence of interferences. Take steps to correct these and reassess Steps 1-3 based on the revised results.

The shape of the platelet histogram was lower than normal and broader. This is consistent with the thrombocytopenia as well as the elevated MPV and PDW.

5. Examine the IPF and compare to the reference interval.

The IPF was elevated above the reference interval, contributing to the elevation of the MPV.

6. Correlate the numerical parameters with platelet morphology.

The platelet morphology identified some large platelets and was consistent with the elevated MPV. The larger platelets can be expected to contribute to the elevated PDW and broader histogram. Larger platelets can also be expected to be younger, consistent with the elevated IPF. Assuming that the rbc count is normal, just one platelet in each field produces an estimate of $20 \times 10^{3} / \mu \mathrm{L}$; this estimate is very close to the automated value.

7. Correlate platelet results with $\mathrm{rbc}$ and wbc parameters and conduct clinical assessment of the patient's values.

In summary, this patient had thrombocytopenia of large, young platelets. The patient had seen an orthopedic specialist due to knee pain. He was prescribed an antiinflammatory drug for the pain due to cartilage injury. Twenty-five days later he returned to his physician with widespread petechial hemorrhages on his lower extremities and the CBC was ordered. Once the patient's medication was changed, his platelet count was within the reference interval three weeks later.

This is an example of a drug-induced immune thrombocytopenia. The peripheral platelet destruction causes thrombocytopenia even as the bone marrow accelerates release of young platelets in compensation. The IPF value was elevated indicating a higher than normal proportion of young platelets due to loss of the older ones from the circulation. Since younger platelets are larger, the MPV was elevated. The PDW was also elevated by the younger larger platelets, causing the histogram to skew to the right.

\section{Case 3.}

A 38-year-old female was referred for a CBC by her internal medicine physician. The results for the platelet parameters are provided in Table 4 and depicted in Figure 4.

\begin{tabular}{lll}
\hline \multicolumn{3}{l}{ Table 4. Platelet Parameter Results for Case 3 } \\
\hline Assay & Patient & Reference Interval \\
\hline Platelet count $\left(\times 10^{3} / \mu \mathrm{L}\right)$ & 116 & $150-450$ \\
MPV (fL) & 9 & $8-12$ \\
PDW (fL) & 11 & $9-15$ \\
Histogram & Refer to & Refer to Figure 4. \\
& Figure 4. & $0.5-7 \%$ \\
IPF (\%) & 3 & Normal \\
Platelet Morphology & Refer to & \\
& Figure 5. &
\end{tabular}

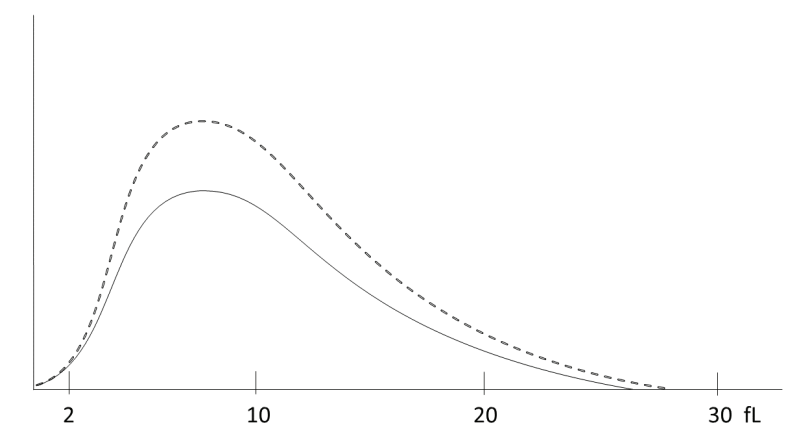

Figure 4. Platelet histogram for case study 3. The dotted line represents a normal histogram. The solid line represents the patient histogram.

1. Interpret the total platelet count relative to the reference interval.

The platelet count was below the reference interval so she has thrombocytopenia.

2. Examine the MPV and compare to the reference interval. 
The MPV was within the reference interval.

3. Examine the PDW and compare to the reference interval.

The PDW was within the reference interval.

4. Examine the platelet distribution histogram for evidence of interferences. Take steps to correct these and reassess Steps 1-3 based on the revised results.

The histogram was of normal shape but lower shorter than normal, consistent with the thrombocytopenia.

5. Examine the IPF and compare to the reference interval.

The IPF was within the reference interval.

6. Correlate the numerical parameters with platelet morphology.

The platelet morphology was normal except that many platelets were surrounding neutrophils (Figure 5). As a result, a platelet estimate was not feasible. Furthermore, it prompted corrective action.

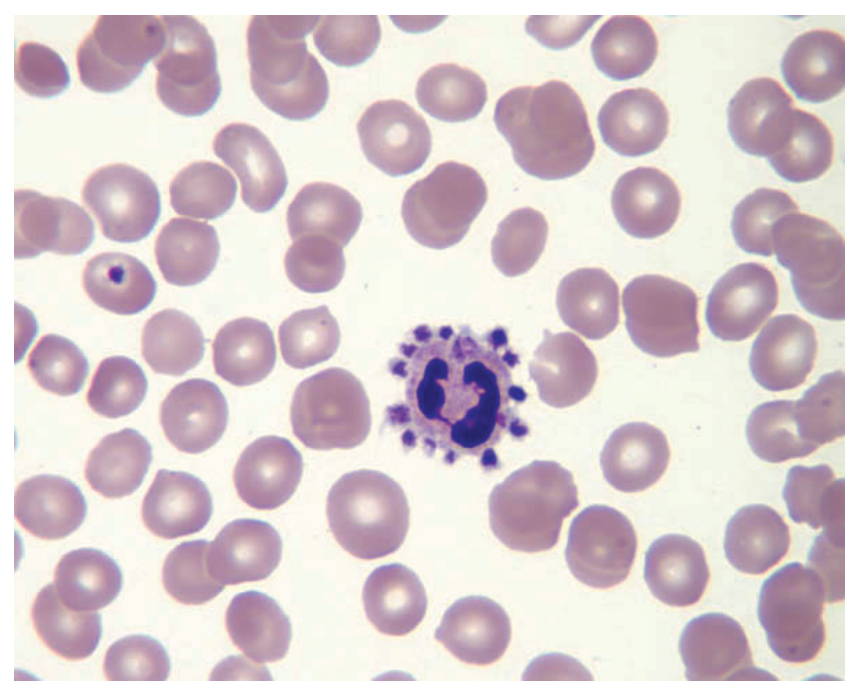

Figure 5. Representative microscopic field for Case 3. (Magnification $\mathrm{X} 1000)$

The patient was requested to return and a new sample was drawn into EDTA but also a sodium citrate tube was drawn. The EDTA sample was tested with results nearly the same as the prior sample, including a low platelet value. Platelet satellitosis was again observed on the blood film prepared from the EDTA sample. The sodium citrate tube was used on the hematology analyzer to generate a platelet count. The resulting platelet value from the instrument was $276 \times 10^{3} / \mu \mathrm{L}$. Since the citrate anticoagulant dilutes the sample, a correction is made to quantitative parameters on citrate samples. The platelet value was multiplied by 1.1 , achieving a final platelet count of $304 \times 10^{3} / \mu \mathrm{L}$. The values for the MPV, PDW, and IPF were unchanged on the citrate sample compared to the EDTA sample. Due to the continuing problem with satellitosis on the blood film, a confirming platelet estimate was not feasible.

With a full set of reliable values, the evaluation steps would be repeated beginning with the platelet count, now showing a value within the reference interval as well as normal indices. These values can then be used for clinical assessment (Step 7).

7. Correlate platelet results with $\mathrm{rbc}$ and wbc parameters and conduct clinical assessment of the patient's values.

Using the revised platelet values, the patient has entirely normal platelets. The wbc and rbc values (not shown) for this patient were also normal. The $\mathrm{CBC}$ was part of an annual screening physical assessment and no abnormalities were expected or found. This case illustrates a case of pseudothrombocytopenia and its detection and correction.

\section{Summary}

Methodical review of the platelet parameters is important to assure that the values are accurate and reportable. The interrelationship among MPV, PDW and IPF mean that changes in one will often mean changes to another. Once the values have been assessed for accuracy, a methodical review ensures that nothing is overlooked as they are evaluated with the $\mathrm{rbc}$ and wbc values for clinical significance.

\section{REFERENCES}

1. Kjeldsberg CR, Swanson J. Platelet satellitism. Blood. 1974; 43:831-6.

2. Maedel L, Doig K. Examination of the peripheral blood film and correlation with the complete blood count. In: Keohane EM, Smith LJ, Walenga JM, editors. Rodak's Hematology: Clinical Principles and Applications. St. Louis: Elsevier. 


\section{FOCUS: INTERPRETING THE COMPLETE BLOOD COUNT}

3 Leader A, Pereg D, Lishner M. Are platelet volume indices of clinical use? A multidisciplinary review. Ann Med. 2012; 44(8):805-16.

4 Kaito K, Otsubo H, Usui N, Yoshida M, Tanno J, Kurihara E, et al. Platelet size deviation width, platelet large cell ratio, and mean platelet volume have sufficient sensitivity and specificity in the diagnosis of immune thrombocytopenia. Br J Haematol. 2005;128(5):698-702.
5 Briggs C, Kunka S, Hart D, Oguni S, Machin SJ. Assessment of an immature platelet fraction (IPF) in peripheral thrombocytopenia. Br J Haematol. 2004;126(1):93-9.

6 Palmer L, Briggs C, Mcfadden S, Zini G, Burthem J, Rozenberg $\mathrm{G}$, et al. ICSH recommendations for the standardization of nomenclature and grading of peripheral blood cell morphological features. Int J Lab Hematol. 2015;37(3):287303. 